\title{
Collective Encoding of Quantum Information in a Quantum Dot Register
}

\author{
A. GrodeckA* And P. Machnikowski \\ Institute of Physics, Wrocław University of Technology \\ Wyb. Wyspiańskiego 27, 50-370 Wrocław, Poland
}

We define the collective logical quantum bit as a state on an array of 2,4 , and 8 quantum dots. We show that such an array can be controlled with less phonon-induced pure dephasing than a single dot. We calculate the dephasing accompanying a single-qubit operation on the collective logical qubit by means of perturbative method describing the open system evolution under arbitrary driving.

PACS numbers: 78.67.Hc, 63.20.Kr, 03.67.Pp

\section{Introduction}

Unique and controllable properties of quantum dots (QDs) together with availability of fast optical control methods indicate that these systems can be considered as attractive candidates for use as the basic units of quantum information - qubits. The physical qubit is defined by relating the logical $|0\rangle$ state to the absence of an exciton in a QD and the logical $|1\rangle$ state to one exciton confined in the dot. The idea of encoding quantum logical values in charge states in QDs $[1,2]$ based on the discrete spectrum of confined carrier states has been recently supported by an experimental demonstration [3].

However, QDs are artificial semiconductor structures and are embedded in a surrounding macroscopic crystal. The interaction of the confined carriers with phonons after optically creating the carrier (exciton) state leads to the loss of coherence on picosecond timescale, which was revealed in optical experiments [4]. This phonon-induced pure dephasing mechanism is explained in terms of the spontaneous relaxation of the lattice after an optically induced change of a confined charge distribution, accompanied by the emission of phonon wave packets $[5,6]$. In real systems the pure dephasing is not the only source of decoherence, an additional impact comes from the finite exciton lifetime (nanosecond timescale). In consequence, in the search for the optimum pulse duration one has to take into

*corresponding author; e-mail: anna.grodecka@pwr.wroc.pl 
account the two different decoherence mechanisms [7]. It has been proposed how to overcome these physical limitations on a QD system: by optimizing the pulse shape $[8,9]$ or by modifying the phonon reservoir [10].

In this paper we propose an alternative approach that overcomes the physical restrictions imposed by dephasing processes on the quantum-logical level. We show that proper design of a QD register may reduce the phonon-induced dephasing, in spite of a larger number of excitons in the array [11]. The idea is to collectively encode the logical qubits in a many-QD system, since collectively acting reservoir on a charge qubit register allows for the subspaces safe against decoherence [12]. This method relies on the fact that the pure dephasing effect is of dynamical non-Markovian character and results only from nonadiabacity of the driving. For a given rate of switching the carrier state only slow phonon modes that cannot follow the evolution of charge distribution are involved, while faster modes follow adiabatically and reversibly. As a result, only a long wavelength part of phonon modes is relevant for the dephasing. These dynamically selected modes may correspond to wavelengths larger than the QD array size, thus allowing for collective interaction and destructive interference of phonon excitations, which leads to a decrease in dephasing.

\section{The system}

We consider an array composed of $N=2^{n}$ quantum dots (physical qubits). Each QD is modelled as a two-level system corresponding to the absence or presence of an exciton with fixed polarization. There is no possibility that phonon-assisted transfers occur, since the wave functions of carriers confined in different dots do not overlap. The Hamiltonian of the system reads

$$
H_{0}=\sum_{l} \epsilon_{l} a_{l}^{\dagger} a_{l}+\sum_{\boldsymbol{k}} \hbar \omega_{\boldsymbol{k}} b_{\boldsymbol{k}}^{\dagger} b_{\boldsymbol{k}}+\sum_{l, \boldsymbol{k}} a_{l}^{\dagger} a_{l} f_{l}(\boldsymbol{k})\left(b_{\boldsymbol{k}}^{\dagger}+b_{-\boldsymbol{k}}\right) .
$$

Here the fermionic operators $a_{l}^{\dagger}\left(a_{l}\right)$ create (anihilate) an exciton with the energy $\epsilon_{l}$ in the $l$-th dot, $b_{k}^{\dagger}, b_{k}$ are bosonic operators for phonon modes with corresponding frequencies $\omega_{k}=c k$. The carrier-phonon coupling constants for an exciton in the $l$-th QD have the symmetry $f_{l}(\boldsymbol{k})=f_{n}^{*}(-\boldsymbol{k})$. The exciton state is described by a product of electron and hole wave functions $\psi_{l}^{(\mathrm{e} / \mathrm{h})}\left(\boldsymbol{r}-\boldsymbol{r}_{l}\right)$, where $\boldsymbol{r}_{l}$ is the dot position. The coupling constants are given by [13]:

$$
f_{l}(\boldsymbol{k})=\sqrt{\frac{\hbar k}{2 \rho_{\mathrm{c}} v c}}\left(\sigma_{\mathrm{e}}-\sigma_{\mathrm{h}}\right) \mathrm{e}^{-\mathrm{i} \boldsymbol{k} \cdot \boldsymbol{r}_{l}} \mathcal{F}_{l}(\boldsymbol{k}),
$$

with the form factors

$$
\mathcal{F}_{l}(\boldsymbol{k})=\frac{1}{\sigma_{\mathrm{e}}-\sigma_{\mathrm{h}}} \int \mathrm{d}^{3} r \mathrm{~d}^{3} r^{\prime}\left|\psi_{l}^{(\mathrm{e})}(\boldsymbol{r})\right|^{2}\left|\psi_{l}^{(\mathrm{h})}\left(\boldsymbol{r}^{\prime}\right)\right|^{2}\left(\sigma_{\mathrm{e}} \mathrm{e}^{-\mathrm{i} \boldsymbol{k} \cdot \boldsymbol{r}}-\sigma_{\mathrm{h}} \mathrm{e}^{-\mathrm{i} \boldsymbol{k} \cdot \boldsymbol{r}^{\prime}}\right) .
$$

Here $\rho_{\mathrm{c}}=5360 \mathrm{~kg} / \mathrm{m}^{3}$ denotes the crystal density, $v$ is the normalization volume of the phonon modes, $c=5150 \mathrm{~m} / \mathrm{s}$ is the sound speed and $\sigma_{\mathrm{e} / \mathrm{h}}\left(\sigma_{\mathrm{e}}-\sigma_{\mathrm{h}}=8 \mathrm{eV}\right)$ 
are the deformation potential constants for electrons and holes (the material parameters correspond to InAs/GaAs system).

The Hamiltonian $H_{0}$ [Eq. (1)] has the structure of the independent boson model [14] and may be diagonalized exactly by means of the Weyl shift operator

$$
\mathcal{W}=\exp \left(\sum_{l, \boldsymbol{k}} a_{l}^{\dagger} a_{l} g_{l}^{*}(\boldsymbol{k}) b_{\boldsymbol{k}}-\text { h.c. }\right),
$$

where $g_{l}(\boldsymbol{k})=f_{l}(\boldsymbol{k}) /\left(\hbar \omega_{\boldsymbol{k}}\right)$. The Hamiltonian $H_{0}$ can now be written in terms of the new operators $\alpha_{l}=\mathcal{W}^{\dagger} a_{l} \mathcal{W}=a_{l} W_{l}$ and $\beta_{\boldsymbol{k}}=\mathcal{W}^{\dagger} b_{\boldsymbol{k}} \mathcal{W}=b_{\boldsymbol{k}}+\sum_{l} \alpha_{l}^{\dagger} \alpha_{l} g_{l}(\boldsymbol{k})$, where $W_{l}=\exp \left[\sum_{\boldsymbol{k}} g_{l}^{*}(\boldsymbol{k}) b_{\boldsymbol{k}}-\right.$ h.c. $]$. The result is

$$
H_{0}=\sum_{l} E_{l} \alpha_{l}^{\dagger} \alpha_{l}+\sum_{\boldsymbol{k}} \hbar \omega_{\boldsymbol{k}} \beta_{\boldsymbol{k}}^{\dagger} \beta_{\boldsymbol{k}},
$$

where $E_{l}=\epsilon_{l}-\sum_{\boldsymbol{k}}\left|f_{l}(\boldsymbol{k})\right|^{2} /\left(\hbar \omega_{\boldsymbol{k}}\right)$. The new operators $\alpha_{l}$ and $\beta_{\boldsymbol{k}}$ represent excitons in the dots surrounded by coherent lattice displacement field (polaron-like quasiparticles) and phonon modes shifted by the presence of a charge distribution.

We assume the availability of a control Hamiltonian of the form

$$
H_{\mathrm{C}}=\frac{1}{2} f(t) \mathrm{e}^{-\mathrm{i} \Delta E t / \hbar} a_{l_{1}}^{\dagger} \ldots a_{l_{N / 2}}^{\dagger} a_{l_{1}^{\prime}} \ldots a_{l_{N / 2}^{\prime}}+\text { h.c. }
$$

which couples the proper pair of states of the $N$-QD register and transfers $N / 2$ excitons from one half of the dots to the other without leaving the subspace spanned by these states. In terms of the redefined operators the control Hamiltonian to the leading order in phonon coupling reads

$$
\begin{aligned}
H_{\mathrm{C}} & =\frac{1}{2} f(t) \mathrm{e}^{-\mathrm{i} \Delta E t / \hbar} \alpha_{l_{1}}^{\dagger} \ldots \alpha_{l_{N / 2}}^{\dagger} \alpha_{l_{1}^{\prime}} \ldots \alpha_{l_{N / 2}^{\prime}} \\
& \times\left[1+\sum_{\boldsymbol{k}} G(\boldsymbol{k})\left(\beta_{-\boldsymbol{k}}-\beta_{\boldsymbol{k}}^{\dagger}\right)\right]+\text { h.c. }
\end{aligned}
$$

with new coupling constants $G(\boldsymbol{k})=\left[g_{l_{1}^{\prime}}(\boldsymbol{k})+\ldots+g_{l_{N / 2}^{\prime}}(\boldsymbol{k})\right]-\left[g_{l_{1}}(\boldsymbol{k})+\ldots+g_{l_{N / 2}}(\boldsymbol{k})\right]$.

The phonon perturbation in Eq. (6) leads to reservoir excitations accompanying any operation on the carrier subsystem. To calculate the effect of the phonon coupling on the driven dynamics we use the second-order (Born) expansion of the evolution equation for the density matrix [7, 15]. The first term in Eq. (6) is included exactly while the second one is treated as a perturbation. The effect of the phonon-induced dephasing on a quantum gate is expressed by the dynamical error, averaged over initial states of the qubit, which may be represented as [7]:

$$
\delta=\int_{0}^{\infty} \mathrm{d} \omega \operatorname{coth}\left(\frac{\hbar \omega}{2 k_{\mathrm{B}} T}\right) \frac{J(\omega)}{\omega^{2}} S(\omega),
$$

where 


$$
J(\omega)=\sum_{\boldsymbol{k}}|G(\boldsymbol{k})|^{2} \omega_{\boldsymbol{k}}^{2} \delta\left(\omega-\omega_{\boldsymbol{k}}\right)
$$

is the spectral density of the phonon reservoir and $S(\omega)$ is defined as follows:

$$
S(\omega)=\frac{1}{12}\left(|F(\omega)|^{2}+|F(-\omega)|^{2}\right) .
$$

Here $F(\omega)$ is a nonlinear spectral characteristics of the driving

$$
F(\omega)=\frac{1}{\hbar} \int_{-\infty}^{\infty} \mathrm{d} t \mathrm{e}^{\mathrm{i} \omega t} f(t) \mathrm{e}^{\mathrm{i} \Phi(t)}
$$

with the rotation angle on the Bloch sphere $\Phi=(1 / \hbar) \int_{-\infty}^{t} f(\tau) \mathrm{d} \tau$. We assume that the operation is performed by a Gaussian control pulse $f(t)=$ $\frac{\hbar \alpha}{\sqrt{2 \pi} \tau_{\mathrm{p}}} \exp \left[-\frac{1}{2}\left(\frac{t}{\tau_{\mathrm{p}}}\right)^{2}\right]$, where $\tau_{\mathrm{p}}$ is the gate duration.

\section{Error reduction by collective encoding}

We consider a linear array of $N=2^{n}$ quantum dots, where the dots are arranged along the $z$ axis as shown in Fig. 1a. The logical collective qubit with $N$ dots is constructed by interchanging occupied and empty dots in the $N / 2$ dot qubit and appending it to the original one. For such a properly designed qubit one finds the coupling constants $G_{n}$ :

$$
G_{n}(\boldsymbol{k})=(2 \mathrm{i})^{n} \prod_{j=-1}^{n-2} \sin \left(2^{j} k_{z} d_{z}\right) G_{0}(\boldsymbol{k})
$$

where

$$
G_{0}(\boldsymbol{k})=\sqrt{\frac{1}{2 \hbar \rho_{\mathrm{c}} v c^{3} k}}\left(\sigma_{\mathrm{e}}-\sigma_{\mathrm{h}}\right) \mathcal{F}(\boldsymbol{k})
$$

is the coupling constant for a single dot placed at the origin and the distance parameter $d_{z}$ is defined in Fig. 1a. The sine function in $G_{n}(\boldsymbol{k})$ indicates that in the long wavelength sector destructive interference occurs, which results in reduction of effective coupling. From Eq. (8) one then finds the phonon spectral densities for the collective qubits in the low-frequency sector

$$
J_{n}(\omega) \approx \frac{2^{n(n-1)}}{2 n+1}\left(\frac{d_{z}}{c}\right)^{2 n} \omega^{2 n} J_{0}(\omega),
$$

where

$$
J_{0}(\omega) \approx \frac{\hbar\left(\sigma_{\mathrm{e}}-\sigma_{\mathrm{h}}\right)^{2}}{4 \pi^{2} \rho_{\mathrm{c}} c^{5}} \omega^{3} .
$$

These spectral densities are plotted in Fig. 1b,c for two sizes of QDs. In both cases, $J_{n}(\omega) \sim \omega^{2 n+3}$ for small $\omega$ and, as a result, for larger collective qubits the spectral density vanishes more quickly for $\omega \rightarrow 0$. This leads to a faster reduction of the pure dephasing error with growing duration of the control pulse. The dynamical error is plotted as a function of the pulse duration in Fig. 2 for two rotation angles of the qubit. 

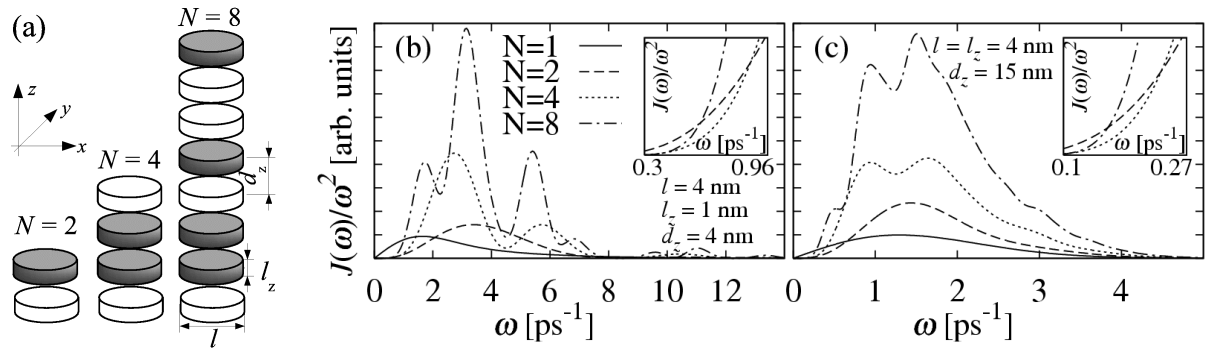

Fig. 1. (a) The collective logical qubit in a linear array of 2, 4, and 8 QDs. Dark dots are occupied in the $|0\rangle$ logical state while the white ones in the $|1\rangle$ state. (b,c) The phonon spectral densities for the physical excitonic qubit (solid line) and for collective qubits composed of $N$ physical qubits (dashed lines) with geometrical parameters as indicated. The insets show the enlarged region of $\omega$ in the range of (b) $0.3-0.96$ and (c) $0.1-0.27 \mathrm{ps}^{-1}$.

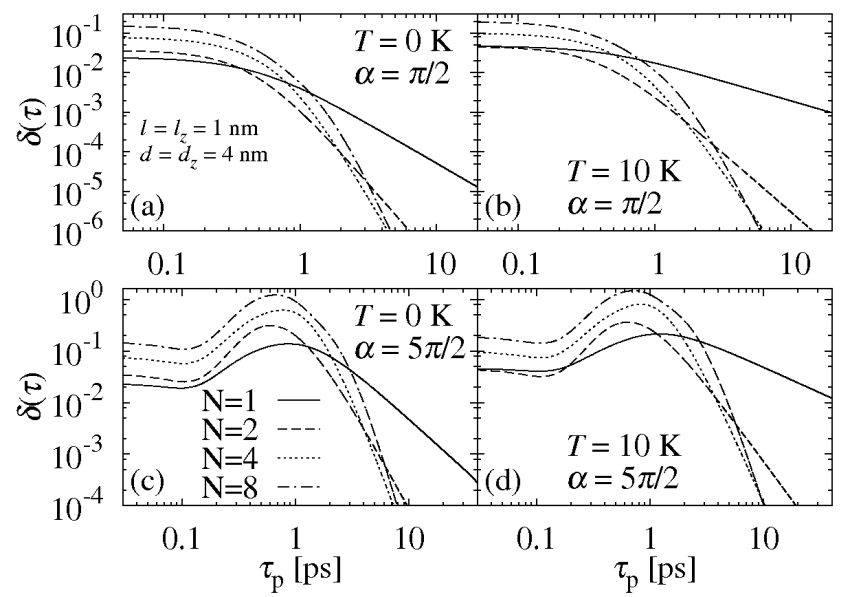

Fig. 2. The phonon-induced dynamical error for a $\pi / 2$ and $5 \pi / 2$ rotation on a physical qubit (solid line) and on a collective qubit with 2, 4, and 8 QDs (lines as shown) with geometrical parameters as indicated.

For the ultrafast pulses error grows with the number of QDs in a register, since in this sector the whole range of phonon modes contributes to dephasing. However, for longer pulses the error for collective qubits is considerably smaller in comparison with the physical qubit. In case of the $5 \pi / 2$ rotation the error dependence on the gate duration is more complicated and the collective logical qubit outperforms the physical one only for longer pulses.

\section{Conclusion}

We have proposed the idea of collective encoding of quantum information in a quantum dot register. The dephasing accompanying a single-qubit operation on 
the collective logical qubit was calculated by means of perturbative method describing the open system evolution under arbitrary driving. We have shown that proper design of an array containing many physical qubits reduces the phonon spectral density in the long wavelength sector and leads to a decrease in the phonon-induced dephasing during an operation on the qubit.

The presented results indicate that the physical limitations resulting from dephasing can be to some extent overcome on the quantum-logical level.

\section{References}

[1] P. Zanardi, F. Rossi, Phys. Rev. Lett. 81, 4752 (1998).

[2] E. Biolatti, R.C. Iotti, P. Zanardi, F. Rossi, Phys. Rev. Lett. 85, 5647 (2000).

[3] X. Li, Y. Wu, D. Steel, D. Gammon, T. Stievater, D. Katzer, D. Park, C. Piermarocchi, L. Sham, Science 301, 809 (2003).

[4] P. Borri, W. Langbein, S. Schneider, U. Woggon, R.L. Sellin, D. Ouyang, D. Bimberg, Phys. Rev. Lett. 87, 157401 (2001).

[5] L. Jacak, P. Machnikowski, J. Krasnyj, P. Zoller, Eur. Phys. J. D 22, 319 (2003).

[6] A. Vagov, V.M. Axt, T. Kuhn, W. Langbein, P. Borri, U. Woggon, Phys. Rev. B 70, 201305 (2004).

[7] R. Alicki, M. Horodecki, P. Horodecki, R. Horodecki, L. Jacak, P. Machnikowski, Phys. Rev. A 70, 010501(R) (2004).

[8] V.M. Axt, P. Machnikowski, T. Kuhn, Phys. Rev. B 71, 155305 (2005).

[9] U. Hohenester, G. Stadler, Phys. Rev. Lett. 92, 196801 (2004).

[10] B. Krummheuer, V.M. Axt, T. Kuhn, Phys. Rev. B 72, 245336 (2005).

[11] A. Grodecka, P. Machnikowski, Phys. Rev. B 73, 125306 (2006).

[12] P. Zanardi, M. Rasetti, Phys. Rev. Lett. 79, 3306 (1997).

[13] A. Grodecka, L. Jacak, P. Machnikowski, K. Roszak, in: Quantum Dots: Research Developments, Ed. P.A. Ling, Nova Science Publ., New York 2005, p. 47.

[14] G.D. Mahan, Many-Particle Physics, Kluwer, New York 2000.

[15] K. Roszak, A. Grodecka, P. Machnikowski, T. Kuhn, Phys. Rev. B 71, 195333 (2005). 